Jakub Ratajczak $^{1 *}$, Adam Sukiennik $^{1 *}$, Sławomir Sielski ${ }^{1}$, Marek Woźnicki ${ }^{1}$, Adrian Klapyta ${ }^{2}$, Jacek Kubica ${ }^{1}$

${ }^{1}$ Department of Cardiology and Internal Medicine, Nicolaus Copernicus University, Collegium Medicum in Bydgoszcz, Poland

${ }^{2}$ Abbott Vascular Structural Heart, Poland

*Jakub Ratajczak and Adam Sukiennik are equal contributors

\title{
Successful reduction of severe mitral regurgitation after implantation of four MitraClip devices
}

\author{
Corresponding author: \\ Adam Sukiennik MD PhD \\ Department of Cardiology \\ University Hospital No. 1 \\ 9 Sklodowskiej-Curie Street \\ 85-094 Bydgoszcz, Poland \\ E-mail: adamsukie@gmail.com \\ Folia Medica Copernicana 2015 \\ Volume 3, Number 4, 176-178 \\ 10.5603/FMC.2015.0014 \\ Copyright (C) 2015 Via Medica \\ ISSN 2300-5432
}

\begin{abstract}
Mitral regurgitation (MR) is one of the most common valvular heart diseases. According to the current guidelines, surgical treatment may be considered in symptomatic patients with severe functional MR. A therapy using the MitralClip (MC) system may be an alternative approach for patients who are at high surgical risk. The presented case report illustrates, as the first one in Poland, a very rare need for the implantation of $4 \mathrm{MC}$ devices in a symptomatic patient with severe functional MR and indicates feasibility and safety of such management.

Key words: mitral regurgitation, MitraClip
\end{abstract}

Folia Medica Copernicana 2015; 3 (4): 176-178

\section{Introduction}

Mitral regurgitation (MR) is one of the most common valvular diseases. Functional or ischemic MR, which results from enlargement and remodeling of the left ventricle (LV), is especially common in patients with chronic heart failure (CHF). According to the current guidelines, surgical treatment may be considered in patients with severe functional MR who remain symptomatic despite optimal medical treatment, with left ventricular ejection fraction (LVEF) higher than $30 \%$ and low comorbidity (Ilb/C) [1]. A therapy using the MitraClip (MC) system may be an alternative approach for patients who are at high surgical risk [2-5]. The MC is a catheter-based system which allows reduction of MR by creating a double-orifice valve after edge-toedge clipping of mitral valve leaflets. The aim of the present paper is to describe the first MC procedure of 4 clips implantation in one patient performed in Poland.

\section{Case report}

A 74-year-old man was admitted to the Department of Cardiology, University Hospital No. 1 in Bydgoszcz at the beginning of December 2015 for the MC proce- dure. The patient had a history of inferior and lateral wall myocardial infarction (MI) in 1995 and coronary artery bypass surgery (CABG) in 1996. Until January 2015 he was treated several times with percutaneous coronary intervention $(\mathrm{PCl})$ and stent implantation. Due to complete atrioventricular block, he had a dual chamber peacemaker (DDD) implanted in 2002, which was upgraded to cardiac resynchronization in 2011. Moreover, the patient had a history of arterial hypertension, chronic kidney disease, gout, nodular goiter, benign prostatic hyperplasia, obesity (body mass index of $31.02 \mathrm{~kg} / \mathrm{m}^{2}$ ) and underwent laparoscopic cholecystectomy. Last year the patient was hospitalized a few times because of exacerbation of $\mathrm{CHF}$ and symptomatic severe MR. In March 2015 he had dialysis due to acute renal failure. After $\mathrm{PCl}$ in January 2015 the patient was on double antiplatelet therapy and was also treated with proton pump inhibitor, statin, beta-blocker, diuretics, angiotensin-converting-enzyme inhibitors, amiodarone, potassium chloride, alpha1 adrenergic receptor antagonist, and levothyroxine. Despite appropriate medical therapy, he stayed in NYHA (New York Heart Association) class III/IV. Transthoracic echocardiography (TTE) performed two months earlier showed dilatation of the left atrium $(L A=58 \mathrm{~mm})$, and dilatation of the right and 
left ventricle $(L V=57 / 65 \mathrm{~mm} ; \mathrm{RV}=32 \mathrm{~mm})$. MR was severe $(4+)$, likely due to the dilatation of LV. Vena contracta, mitral valve leaflets coaptation depth and length were $8 \mathrm{~mm}, 7 \mathrm{~mm}$ and $4 \mathrm{~mm}$, respectively. Length of the anterior leaflet was $25 \mathrm{~mm}$ and posterior $15 \mathrm{~mm}$. Echocardiography also revealed small/medium tricuspid regurgitation and elevated to $46 \mathrm{~mm} \mathrm{Hg}$ pulmonary artery systolic pressure. Myocardial contractility was impaired, with LVEF 35\% due to akinesia of the basal and medial segments of the lateral wall, akinesia of the apical and medial segments of the inferior wall and hypokinesis of the interventricular septum and basal segment of the inferior wall. Transesophageal echocardiography (TEE) performed on the same day confirmed the severity of MR with vena contracta $8 \mathrm{~mm}$ (Fig. 1), mitral valve leaflets coaptation depth and length of $9 \mathrm{~mm}, 10 \mathrm{~mm}$ and $4 \mathrm{~mm}$ respectively. The length of the anterior leaflet was $30 \mathrm{~mm}$ and the posterior $17 \mathrm{~mm}$. There was at least double-flowed jet of regurgitation in the intercommissural view, with vena contracta $8 \mathrm{~mm}$ for each jet. In the left atrial appendage, an inhomogeneous echo $(10 \times 4 \mathrm{~mm})$ was seen. Coronary angiography showed multivessel disease with borderline left main distal stenosis, occlusions of the mid left anterior descending artery (LAD), proximal circumflex (Cx) artery and proximal right coronary artery (RCA). There were also: a patent arterial graft (the left internal mammary artery to the LAD), an occluded saphenous venous graft (SVG) to the RCA and a patent SVG to the obtuse margin and distal Cx. After the Heart Team discussion, the patient was disqualified from the surgery, due to the poor clinical condition and high surgical risk (EuroSCORE $\|=10.69 \%$ ), and a decision to perform the MitraClip procedure was made. The procedure was performed under general anesthesia, with the angiographic and TEE guidance. After the right femoral vein puncture, the transatrial puncture was performed in the precise, prespecified location. The MitraClip system was then introduced to the left atrium and, after careful navigation, the clip

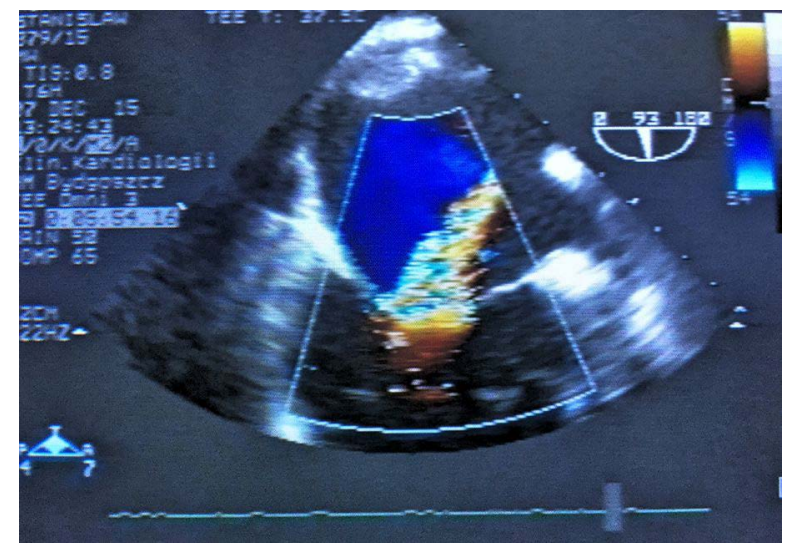

Figure 1. Baseline, grade 4, mitral regurgitation in TEE was introduced to the LV cavity (Fig. 2). Next, the mitral valve leaflets were grasped in the most medial location of the regurgitant jet. In the TEE, the mean pressure gradient (MPG) was $2.6 \mathrm{~mm} \mathrm{Hg}$ and the mitral valve area (MVA) calculated from pressure half-time (PHT) was $2.7 \mathrm{~cm}^{2}$. The decision to implant another clip was made due to severe 4+ lateral regurgitant jet (Fig. 3). After the second clip implantation, just lateral to the first one, the MPG was $2.9 \mathrm{~mm} \mathrm{Hg}$ and the MVA calculated from PHT was $2.6-2.7 \mathrm{~cm}^{2}$ in the TEE. There was also moderate $(3+)$ lateral regurgitant jet with vena contracta $6 \mathrm{~mm}$ in the TEE. The interventional team made a decision to implant next clip, lateral to the implanted ones. After that MPG increased to $3.5 \mathrm{~mm}$ $\mathrm{Hg}$ and the MVA calculated from PHT decreased to $2.5 \mathrm{~cm}^{2}$. Unfortunately, lateral regurgitant jet persisted moderate $(3+)$, with vena contracta $0.6 \mathrm{~cm}$. The MR severity was reduced to $2+$ (Fig. 4), with MPG $4.0 \mathrm{~mm}$ $\mathrm{Hg}$ and the MVA calculated from PHT $2.5 \mathrm{~cm}^{2}$ after implantation of four MC (Fig. 5). The patient was hemodynamically stable and we finished the procedure without any complications. The femoral vein access was protected with suture and pressure dressing.

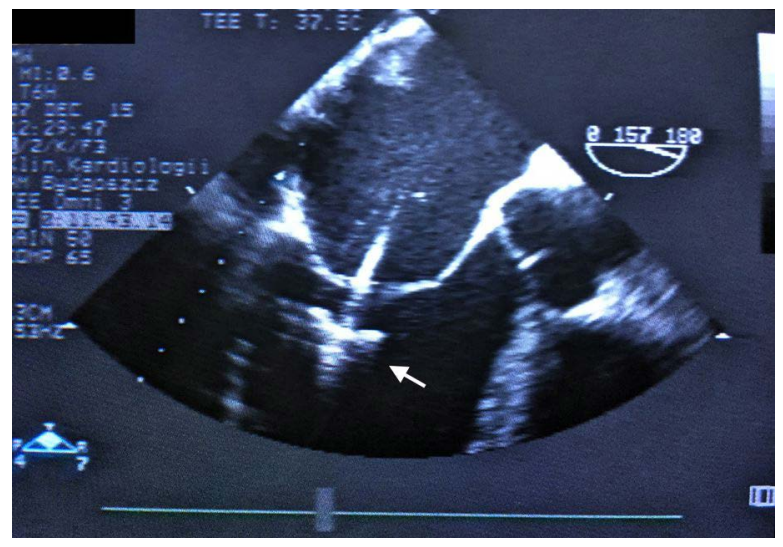

Figure 2. MitraClip (arrow) in the LV cavity before grasping of the mitral leaflets

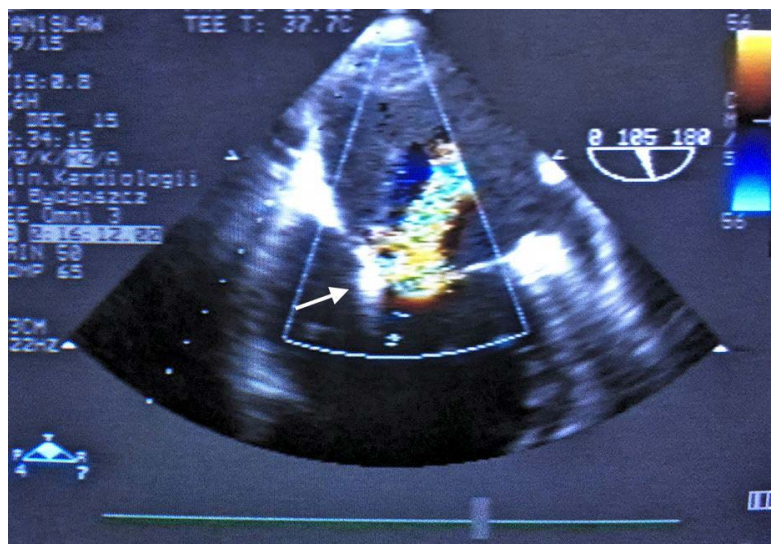

Figure 3. TEE after implantation of the first MitraClip (arrow) 


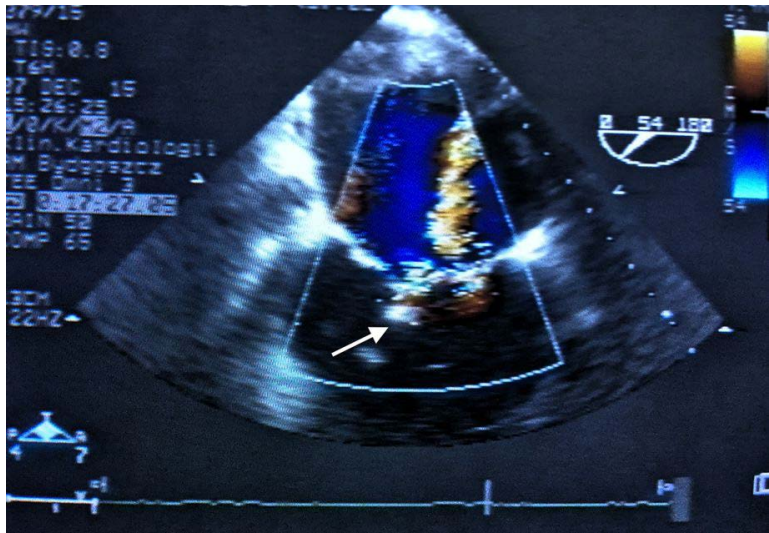

Figure 4. Final, grade 2, mitral regurgitation in TEE after implantation of four MitraClips (arrow — only one MitraClip visualized in this view)

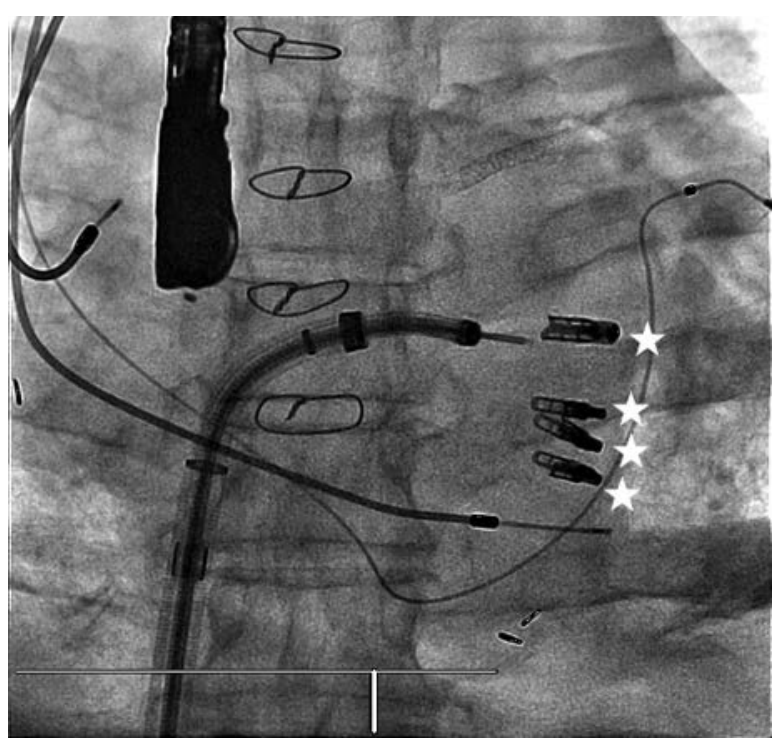

Figure 5. Final fluoroscopy after implantation of four MitraClips (asterisks)

In the control TTE four hours after the procedure, there was a slight increase in MR to $2+/ 3+$, probably due to different examination condition of the patient.

\section{Discussion}

Catheter-based repair of the mitral valve using MC therapy may be an alternative to surgery for patients with high risk of operation due to severe, symptomatic, functional MR, prior MI, high NYHA class, decreased LVEF and with a history of cardiac surgery. The EVEREST II study [6] (Endovascular Valve Edge-to-Edge Repair Study) showed that MC is less effective in reducing MR than conventional surgery, although it proved superior safety of $\mathrm{MC}$ procedure and comparable improvements of NYHA class and quality of life. MC therapy turned out to be a valuable option also for critically ill patients who would persistently need inotropes or who could not be weaned from a ventilator [7]. The described procedure is unique because, to the best of our knowledge, this is the first case in Poland that four clips were implanted during the treatment using the MC system. Reported cases of the implantation of four or more clips are very rare in the literature. In the ACCESS-EU registry [8], 3 or more devices were implanted in $<3 \%$ of patients. In a study by Koifman et al. [3] and French registry [5], none of the patients had more than 2 clips implanted. A registry from Switzerland [4] also showed that in none of the cases 4 clips were used and only two patients (3\%) received 3 clips. In a study by Franzen et al. [9], the highest number of implanted devices was 3 in one patient $(2 \%)$. Four clips were implanted only in one patient (3\%) in a study by Gaemperli et al. [10].

\section{Conclusions}

The presented case report illustrates, as the first one in Poland, a very rare need for implantation of $4 \mathrm{MC}$ devices in a symptomatic patient with severe functional MR and indicates feasibility and safety of such management.

\section{References}

1. Vahanian A, Alfieri O, Andreotti F et al. Guidelines on the management of valvular heart disease (version 2012) The Joint Task Force on the Management of Valvular Heart Disease of the European Society of Cardiology (ESC) and the European Association for Cardio-Thoracic Surgery (EACTS). Eur Heart J 2012; 33: 2451-2496.

2. Munkholm-Larsen $S$, Wan B, Tian D H et al. A systematic review on the safety and efficacy of percutaneous edge-to-edge mitral valve repair with the MitraClip System for high surgical risk candidates. Heart 2014; 100: 473-478.

3. Koifman E, Fefer P, Hay I, Feinberg M, Maor E, Guetta V. MitraClip implantation for high risk patients with severe mitral regurgitation: the Sheba experience. Isr Med Assoc J 2014; 16: 91-95.

4. Toggweiler S, Zuber M, Sürder D et al. Two-year outcomes after percutaneous mitral valve repair with the MitraClip system: durability of the procedure and predictors of outcome. Open Heart 2014; 1: e000056.

5. Armoiry X, Brochetd E, Lefevre T et al. Initial French experience of percutaneous mitral valve repair with the MitraClip: A multicentre national registry. Arch Cardiovasc Dis 2013; 106: 287-294.

6. Feldman T, Foster E, Glower DD et al. Percutaneous repair or surgery for mitral regurgitation. N Engl J Med 2011; 364: 1395-1406.

7. Pleger ST, Chorianopoulos E, Krumsdorf U, Katus HA, Bekeredjian R. Percutaneous edge-to-edge repair of mitral regurgitation as a bail-out strategy in Critically III Patients. J Invasive Cardiol 2013; 25: 69-72.

8. Maisano $F$, Franzen $O$, Baldus $S$ et al. Percutaneous mitral valve interventions in the real world: early and 1-year results from the ACCESS-EU, a prospective, multicenter, nonrandomized post-approval study of the MitraClip therapy in Europe. J Am Coll Cardiol 2013; 62: 1052-1061

9. Franzen O, van der Heyden J, Baldus S et al. MitraClip therapy in patients with end-stage systolic heart failure. Eur J Heart Fail 2011; 13: 569-576.

10. Gaemperli O, Biaggi P, Gugelmann R et al. Real-time left ventricular pressure-volume loops during percutaneous mitral valve repair with the MitraClip system. Circulation 2013; 127: 1018-1027. 